\title{
Significados y percepciones del ambiente por adolescentes: Reflexión en tiempos de pandemia.
}

\author{
Juan Camilo Cardona Castaño ${ }^{1}$ \\ Martha Custodia Lamprea Zona ${ }^{2}$ \\ Flor Aida Cubides Suárez ${ }^{3}$
}

\section{Información de artículo:}

Recibido: 30/09/2021

Aprobado: 15/12/2021

\section{Palabras claves:}

COVID 19; Educación Ambiental; Percepciones Ambientales; Significado del Ambiente;

\section{Resumen}

Las percepciones de algunos adolescentes sobre el ambiente, es factor esencial dentro de la comprensión hombre - naturaleza. La actual pandemia producida por el COVID-19, permitió que los sujetos, pudieren observar su entorno con detenimiento, conduciendo a cambios en las percepciones. Todo ello, se construyó desde la educación ambiental; herramienta que sirvió para explorar y sentir los beneficios del espacio, todo ello durante el confinamiento. El objetivo de la investigación, consistió en analizar e interpretar las percepciones que tienen un grupo de adolescentes, en situación de vulnerabilidad frente al entorno en tiempos de confinamiento por la pandemia COVID-19. La investigación está situada en el paradigma cualitativo de tipo narrativo, por medio de dibujos, entrevistas y técnica de grupo focal, se desarrolló la investigación. Los hallazgos: el ambiente como un todo. Los procesos urbanos y el ruido de la ciudad no permiten develar la importancia de la naturaleza. No obstante, el ambiente como una estructura inquebrantable ante la realidad humana, el silencio que produjo la pandemia condujo al grupo de estudio a nuevas experiencias dentro del espacio que habitan, por ejemplo: sonidos, observación de nuevas aves, insectos, etc. En finalidad, los participantes reflexionaron sobre los cambios que trajo el confinamiento y el significado del ambiente estrechando la relación con la naturaleza. También, el crecimiento de la ciudad trae perturbaciones en la naturaleza, cambiando el paisaje.

\section{Keywords:}

COVID-19; Environmental education; Environmental perceptions; Meaning of the environment

\footnotetext{
1 Consultor Ambiental Y Administrador Ambiental de la Universidad Santo Tomás, adscrito al grupo de investigación en educación en Enfermería. (iD) https://orcid.org/oooo-0002-9631-9870

2 Máster en Ciencias Ambientales. Profesora de la Universidad Santo Tomás. (D): https://orcid.org/oooo-00o2-8173-9477

3 Especialista en Gestión de Proyecto Sociales. Coordinadora del Centro de Atención Universitaria de la Universitaria Santo Tomás. (D): https://orcid.org/oooo-0002-3540-086X,
}

\footnotetext{
Cardona-Castaño, J. C., Lamprea-Zona, M. C., \& Cubides-Suárez, F. A. (2021). Significados y percepciones del ambiente por adolescentes: Reflexión en tiempos de pandemia. Revista Electrónica De Conocimientos, Saberes Y Prácticas, 4(2), 9-24. https://doi.org/10.5377/recsp.v4i2.13385
} 


\section{Meanings and perceptions of the environment for adolescents: Reflection in times of pandemic.}

\section{Abstract}

The perceptions of some adolescents about the environment is an essential factor in understanding man - nature. The current pandemic produced by COVID-19, allowed the subjects to observe their surroundings carefully, leading to changes in perceptions. All of this was built from environmental education; tool that served to explore and feel the benefits of space, all during confinement. The objective of the research was to analyze and interpret the perceptions that a group of adolescents has, in a situation of vulnerability to the environment in times of confinement due to the COVID-19 pandemic. The research is located in the qualitative paradigm of a narrative type, through drawings, interviews and a focus group technique, the research was developed. The findings: the environment as a whole. Urban processes and city noise do not allow us to reveal the importance of nature. However, the environment as an unbreakable structure in the face of human reality, the silence that the pandemic produced led the study group to new experiences within the space they inhabit, for example: sounds, observation of new birds, insects, etc. Finally, the participants reflected on the changes brought by confinement and the meaning of the environment, strengthening the relationship with nature. In addition, the growth of the city brings disturbances in nature, changing the landscape.

\section{Introducción}

El presente artículo reflexiona desde la educación ambiental, sobre las percepciones del individuo, frente al espacio que habitan y como logran construir un referente del ambiente; a través de la experiencia y el significado que el entorno les provee a los adolescentes.

La investigación se desarrolló en medio de la actual crisis de salud pública ocasionada por el COVID-19, en una fundación sin ánimo de lucro que atiende niños, niñas y adolescentes en proceso de restitución de derechos. La fundación brinda educación a grupos de beneficiarios en situación de vulnerabilidad, en algunas sedes los adolescentes viven internados, como es el grupo focal estudiado. La institución es descentralizada del sistema educativo colombiano, lo cual permite el abordaje de nuevas problemáticas en los diversos contextos pedagógicos.

Es así, como aparece la educación ambiental en una fundación que opera como actor no centralizado del sistema educativo colombiano, garantizando el aprendizaje pleno sobre el ambiente. De tal forma, la investigación se centró en comprender como los jóvenes perciben y entienden el significado del ambiente en medio de la crisis mundial 
de salud pública. Si bien, la actual crisis está llevando hacer nuevas apuestas en el ámbito educativo no inmerso dentro del sistema educativo convencional colombiano, también emergen realidades en la conceptualización de áreas del conocimiento que resultan importantes para el análisis, dada su complejidad; forma y tiempo.

La investigación solo tuvo una única etapa, cuyo objetivo fue interpretar y analizar las relaciones del sujeto con el espacio habitado, antes, durante y después de la pandemia COVID-19, donde los adolescentes por medio de narrativas e imágenes respondieron y mostraron sus planteamientos de percepciones del contexto, frente al problema de salud pública mundial y su interrelación con el ambiente.

La investigación cualitativa de tipo narrativa, cuyo trabajo se realizó con adolescentes, en forma de grupo focal. Lo anterior permitió, formalizar un dialogo y construir una narrativa sobre cómo es vivir durante la pandemia COVID-19 teniendo el entorno como factor de interacción y el significado del ambiente que surge tras el confinamiento. El proyecto inicia cuando Colombia tenía 10.000 mil infectados y termina cuando llega aproximadamente a los 30.000 mil casos reportados por la infección COVID-19. Esto supone una realidad en la forma de vida y el entendimiento de la pandemia, pero cobrando profundas reflexiones sobre el ambiente antes, durante y muy posiblemente después de dicha emergencia de salud pública.

De tal modo, que los participantes desarrollaron ideas que fusionaban el significado del ambiente, la crisis actual y la generación de hipótesis a priori que dinamizaban el proceso de interacción en el área de estudio. Por ello, se hicieron talleres de educación ambiental, para que los jóvenes configuraran unas respuestas y desarrollaran un constructo frente a las preguntas detonantes, por ejemplo: ¿Cómo está mi espacio por efectos de la cuarentena?, ¿Qué cambios percibo en el ambiente durante la cuarentena? y ¿Cómo me relaciono con el espacio en el que vivo?; Las anteriores preguntas, llevaron a hipotetizar durante el proceso práxico; la realidad del mundo actual, por el cual se está atravesando, siendo esta una respuesta del ambiente, ante los actos antrópicos. También, falta más entendimiento del entorno y el espacio que el hombre habita, promoviendo respuesta de respeto para con la naturaleza.

De igual modo, se debate como el significado del ambiente y el entorno, estado a cambios de la percepción; dado que la actual crisis sanitaria llevó a un prolongado aislamiento de las comunidades, donde pareciera que en algunos espacios del ambiente estuvieron a salvo del caos de la ciudad o en una relativa calma. No obstante, La situación actual conllevó a implementar un trabajo que buscó, analizar la percepción de los adolescentes, en cuanto al ambiente como un macrosistema que se recupera a causas de la cuarentena, (Osuna, 2021; Bracho, 2021). Asimismo, dentro del grupo focal se disertó como el crecimiento de los ambientes impactados por factores antrópicos, evidencia la relación del entorno y el sujeto, Leff,(1998). Dicha premisa, permite en 
tiempos de la pandemia COVID19 hacer una profunda búsqueda, sobre la interacción de un grupo de personas frente al espacio habitado (Leff, 2004; Díaz, Cruz, 2020).

\section{Literatura}

De tal modo, que la educación ambiental como estrategia, permitió indagar sobre las percepciones del ambiente dada por los jóvenes, en condición vulnerable. Dejando al descubierto, sus ideas y criterios sobre la actualidad y como el mundo viene en un proceso de aislamiento y cuarentena, así como la relación frente a la disminución y reducción de los daños e impactos ambientales en el planeta, por lo menos en estos pocos días donde la oportunidad fue: la forma de evitar la sobre explotación de los recursos naturales no renovables, Abril, (2021). Por ello, dicha situación de salud pública deja muchas lecciones de lo humano en el significado del ambiente, entorno y la configuración del fortalecimiento del sujeto en las dinámicas sociales y culturales en pro del cuidado ambiental, (Arias, Rincón, 2010).

De acuerdo con, Rosales, (2018) las percepciones son ideas creadas por el individuo, desde la experiencia, la práctica, la vivencia y la forma de interactuar con los elementos que hacen parte de su aprendizaje. Expuesto lo anterior, las prácticas de educación ambiental, contribuyen a una serie de cuestionamientos sobre el espacio, aun cuando la observación sobre el mismo devela cambios, sentimientos y formas de interpretaciones distintas, (Abril, 2015; Casuro, 2018).

De ahí que, la comprensión del sujeto, significado y percepción del ambiente promueven la necesidad de nuevos significados de la interacción con el entorno, aún más cuando en confinamiento se requiere estar. En consecuencia, la reconstrucción del espacio, con miradas más reflexivas, llevaron al sujeto actor a la problematización sobre el cuidado de la naturaleza. En reciprocidad la naturaleza es dadora de bienestar al sujeto, abriendo una interrelación de las realidades sociales y ambientales frente al cambio de vida por una situación de coyuntura dentro del entorno, Leff, (1998); Pereira, (2010). Las prácticas educativas ambientales, promueven un cambio en la construcción del sujeto que hace parte de un inmerso sistema de vida transitando desde lo humano irracional y lo humano racional, facilitando un descubrimiento de las ideas, los significados y sus propias percepciones que tiene frente al ambiente, (Quintero, Solarte, 2019).

De acuerdo a lo anterior, la complejidad del significado del ambiente en la actualidad está enmarcada dentro del desarrollo sostenible se demuestra a través de acciones y modelos de optimización que todos los sistemas implementan e incluso el educativo, bien sea dentro o fuera de un subsistema, con el mismo principio, ahondar sobre las necesidades que el ambiente tiene y la interacción con el sujeto, (Castro, 2004; Leff, 2007; Rodríguez, 2010). Con relación a lo anterior, es un paso del pensamiento antrópico; sentir y dialogar con lo natural en una época, donde estar aislado 
y/o en confinamiento se volvió una constante progresiva, que parece sin retorno, (Fernández, 2008; Arias, 2021).

Asimismo, la perspectiva social del entorno construido por el hombre cambió por las percepciones del sujeto, Espino, Olaguez, Davison, (2015). Lo anterior, genera una fuerte transformación dentro de las interacciones de lo humano con lo ecológico, de lo pragmático con lo reflexivo frente a variaciones relacionadas con el medio ambiente y sus componentes, (Esteban, 2015).

\section{- Concepto de percepción}

Desde lo filosófico, el concepto se articula con el entendimiento de las realidades a las que el sujeto está expuesto, Arias (2006). La percepción está intrínseca en la configuración del juicio sobre los actos o cosas, que existen en las acciones humanas, o lo que proporciona el entorno, Hernández, (2016). La percepción, no tiene limitantes en la interpretación del mundo externo, los acontecimientos físicos, naturales, sociales, en general todo aquello que permite una disertación de la experiencia de vida, que tiene como resultado un juicio inminente sobre la realidad entendiéndose la realidad: como un constructo perceptual, (Rosales-Sánchez, 2018).

\section{- Educación Ambiental}

Es considerada como herramienta poderosa en escenarios, donde se debe articular los procesos de entendimiento y la complejidad de la problemática ambiental. La educación ambiental desde la práxis y el discurso, permiten la comprensión de cualquier acontecimiento que se desarrolla en el entorno e impacte en lo humano, para ser explicado desde lo pedagógico, asimismo; hacer que el sujeto de aprendizaje brinde un significado a lo que parece insignificante y logre recopilar las experiencias tangibles o intangibles que el ambiente como proveedor de bienestar, da a la especie humana, (Eschenhagen, 2007; Martínez, 2010; Martínez, 2014; Montoya,2019).

\section{- Significado del ambiente}

El ambiente, como una construcción humana o también como algo natural que florece por efectos de la evolución, dicho significado no solo debe ser tratado como algo meramente biológico también desde lo psicológico y lo social; de tal modo, que el ambiente, se vuelve el sistema proveedor de bienestar que está en paralelo con los recursos naturales, Edel, Ramírez, (2006). Es importante aclarar, que el significado de ambiente, debe ser analizado desde lo cultural como un fenómeno lingüístico que responde a las necesidades que una especie tiene y son resueltas por el ecosistema o el medio natural con el que se resuelven, desde una visión más popular pero no menos importante, es todo lo que rodea al hombre, resultando ser una construcción del 
interaccionismo hombre naturaleza fundamental en la base del desarrollo humano y demás especies, (Morales, 2016).

\section{Metodología de la investigación}

La investigación se encuentra en el paradigma cualitativo de tipo narrativo. La narración en investigación, genera constructos literarios que conllevan al análisis y la disertación del fenómeno por medio de creaciones textuales, imágenes y elementos sonoros, dando respuesta al fenómeno tratado, Domínguez, Herrera, (2011). Por otra parte, la narrativa permite el análisis de la experiencia de vida y su relación de experimentación con el entorno por medio de relatos, (Aguirre, 2012). Por ello, el objetivo de esta investigación radica en analizar e interpretar las percepciones que tienen un grupo de adolescentes, en situación de vulnerabilidad frente al entorno en tiempos de confinamiento por la pandemia COVID-19.

La investigación se realizó en 26 participantes, de una organización sin ánimo de lucro que rehabilita jóvenes; de igual modo, se analizaron tres categorías: percepciones sobre el espacio habitado, ambiente y el sujeto, pandemia y ambiente; de ahí que los participantes hicieron una interpretación a través de dibujos y constructos conceptuales sobre el significado del ambiente.

El instrumento para recolectar la información fue la entrevista, está estuvo dividida en tres categorías: primera, percepciones sobre el espacio habitado: desde esta unidad de análisis, buscando saber cómo los adolescentes interpretaban el espacio que habitaban y las necesidades que se encuentran en él. Segundo, ambiente y el sujeto: buscaba comprender como los participantes interactúan con el medio ambiente y como era su relación con el mismo. Tercero, pandemia y ambiente: Relación de la pandemia con el medio natural, posible causa- efecto.

La observación fue de tipo pasiva, es decir: el investigador no hace juicios de valor, ni punitivos frente a los fenómenos encontrados. De acuerdo con, Rekalde, Vizcarra, Macazaga, (2014), dicho tipo de observación permite al investigador la observación de sucesos en el escenario o lugar que se investiga, siendo fundamental para determinar interacciones del sujeto con el espacio y sus componentes.

La técnica de análisis fue la técnica grupo focal: permite una construcción de diálogos entre participantes, debate e ideas presentes en el sujeto, García-Huidobro, (2016). Permitió a la investigación fortalecer la construcción de experiencias vividas en la pandemia y el encuentro con el ambiente. Se usó diario de campo, que permitió el registro de acontecimientos presentes, así como el proceso de la investigación. De igual modo, el análisis fue producto de 3 entrevistas directas no estructuradas, con preguntas detonantes. 


\section{Resultados}

\section{- Pandemia y Ambiente}

Esta unidad de análisis se construyó con el fin de saber y entender como los participantes estaban interactuando con el entorno y sus realidades sociales, ambientales y adaptativas frente a la pandemia, aporta, participante 1.

"Vivimos en un espacio con contaminación, ahora con la pandemia no podemos salir, pero ha sido normal para nosotros porque siempre hemos estado aquí, pero lo que percibo: han llegado mayores pajaritos y no se oye tanto ruido" Participante 1.

Lo anterior, demuestra que el sujeto está viviendo en el ambiente impactado, por ello deja una fuerte reflexión sobre el medio natural, a través de su propia experiencia. La percepción del espacio habitado, es descrito por el participante a través del entorno, como algo favorable, en cuanto al significado del ambiente, una comunión del sujeto con los componentes naturales. Aporta el participante 3.

“Tengo entendido que esto hace unos años, era rural, pero ahora somos más pequeños, sino miren ese edificio de 15 pisos que tenemos diagonal casi que desde allí nos pueden vigilar. Pero como todo mundo anda encerrado, nadie se asoma a mirarnos" Participante 3 .

De acuerdo con lo anterior participación, se puede analizar lo siguiente: primero, no se distingue lo rural de lo urbano, debido a que las fronteras fueron desligadas por los procesos económicos y urbanísticos. Donde el significado de estar al lado de lo urbano o lo rural no tiene mayor relevancia, tal vez sea porque nunca se conoció la diferencia. Pero en tiempos del confinamiento surge la pregunta ¿dónde inicia lo urbano y lo rural, dentro del espacio? Aporta el participante 2.

"No hemos sentido la pandemia, pues estamos aislados en una zona que es medio rural, entonces vivir así, nos hace adaptarnos a los problemas de salud que hay afuera. Antes había mucho polvo por la construcción ese edificio, en esta época de confinamiento el humo y el polvo de los carros ha disminuido" Participante 2.

La respuesta transmite una preocupación, el desplazamiento de la intimidad de su entorno causado por factores externos. Reconoce que estar relativamente lejos de lo urbano es importante para no estar expuesto al problema de salud pública existente. De tal modo, la integración del sujeto con el sistema ambiental es inherente, generando respuestas y visiones críticas, porque la dinámica del ambiente está enmarcada en la diversidad social, personal y de los ecosistemas. Se puede decir que la percepción sobre ambiente, es definida como aquel equilibrio y bienestar para el humano (Leff, 2018). 
Asimismo, la relación del individuo con el medio natural dentro de la emergencia de salud pública, viene cobrando una elevada reflexión, lo cual es altamente emergente en el sentido que se valora los recursos naturales, desde lo sistémico e integrado de forma dinámica e interactiva con el espacio, Plachot, (2021). Cuyo, entendimiento de la recuperación del ambiente para los participantes sigue siendo un entramado robusto con los patrones de comportamientos favorables. No diferente, el participante 2. Considera:

"Yo considero, que el ambiente está totalmente sano y limpio, creo que es por esta situación. Lo que estamos viviendo" Participante 2.

Con relación a la anterior afirmación, se demuestra que se percibe un cambio del significado social sobre el ambiente, dado por la sociedad misma. Por ello, no se aleja de la oportunidad de entender la transformación del espacio habitado, ya que es resiliente, con capacidad variada de respuesta, donde el sujeto observa un imaginario llamado entorno que surgen a través de los cambios y cosas que son nuevas dentro del contexto.

\section{- Ambiente y el sujeto}

El grupo de participantes, manifestó un gran interés por explicar a través de dibujos y dialogo continuo, el fenómeno del ambiente y la pandemia, siendo de suponer, que para aquellos participantes estar aislados es natural debido a su estatus de penalidad y de infractores, pero esto no hace que el sistema que compone el contexto sea meramente adinámico, más bien, esta es una situación de coyuntura que pone a reflexionar al humano encontrando un significado de relación entre el entorno, paisaje y aquellos sonidos que se perciben en medio del silencio que produce la cuarentena. Los cambios generados por dicha situación se ven reflejados en el desenvolvimiento de la vida diaria, también en la disminución y la reducción del consumo de los recursos naturales que paulatinamente se van agotando. De tal forma, el participante número 1 Consideró, lo siguiente:

"La cuarentena nos ha llevado a escuchar, ver y sentir cosas nuevas y como estamos en lo rural mucho más.... pues yo considero que este sitio siempre fue cálido y ameno, el ruido no falta.... pero ahora es mínimo y esto me ha permitido ver especies de nuevas aves, ver flores que quizá no estaban o tal vez nunca las veía." Participante 1.

Lo que aporta el participante, resulta comprensible, la explicación: el sujeto está inmerso en un mar de información la cual, no permite una observación activa del espacio, asimismo, la interacción hombre-naturaleza viene fallando, más por la imposición de los sistemas económicos e incluso humanos que se vienen instaurando en el espacio, dejando de lado, lo emergente, es decir: lo que está allí y siempre habita el ambiente, pero es nuevo para el individuo. 


\section{- Percepciones sobre el espacio habitado}

La relación hombre-naturaleza, fundamental en el desarrollo evolutivo, adaptativo, social y de bienestar, Quintero, Solarte, (2019). Por ello, la interacción del sujeto como un actor dentro del medio natural es la base dentro del análisis de lo ambiental, esto permite considerar varias cosas: el espacio habitado como un nicho adaptado a las necesidades humanas, (Morin, 2001). En particular, el dialogo del sujeto con el medio natural garantiza un sistema de supervivencia, pero esto último está en riesgo, debido a procesos neoliberales y de sobre explotación de los recursos naturales, sin permitir una recuperación del espacio explotado, (Novo, Murga, 2010,).

Por ello, el análisis de la relación del hombre con el entorno, supone una adecuación del mismo para que sea adaptable a sus propias necesidades. Con todo ello, los participantes consideraron que el espacio que ellos habitan es un conjunto de situaciones, por ejemplo: no decidieron estar internados en la fundación. Sin embargo, reconocen que es importante cuidar el ecosistema y hacer un adecuado uso de los recursos naturales, como lo son: agua, paisaje, fauna, flora y suelo, puesto que estos componentes tienen muchas presiones por las condiciones económicas y de mercado impartidas por el sistema productivo de Colombia y la región. De tal modo, aporta el participante 2 .

"Yo cuido el ambiente, separando y no tirando basuras al suelo. Revuelvo la compostera, para mí es un buen cuidado........."

Investigador: ¿Qué tiene que ver el compostaje?

Participante: "la sociedad solo nos enseña a consumir, pero no a cambiar o transformar lo que generamos, por eso cambiar la forma de cuidado y de mejoramiento de lo que le hacemos al ambiente, depende de cada uno de nosotros" Participante 2.

Con relación a la anterior respuesta, la sociedad es generadora de cambios al nivel del ambiente, porque está englobada en el sistema económico, haciendo que las medidas para cuidar al ambiente se vuelvan sin importancia. Siendo, la estructura social no proveedora de un cambio de forma positiva, además no permite un dialogo directo y ameno con el entorno, (Leff, 2006). El participante 2. Indirectamente deja al desnudo que las soluciones pertenecen al sistema social y económico neoliberal y a la complejidad humana tan subjetiva. Es pertinente mencionar, que el sistema de mercado no sitúa limite, y no advierte sobre soluciones con respecto al daño y la perturbación a los ecosistemas, espacios y territorios. Si bien, en la actualidad los modelos económicos están optando por del desarrollo sostenible promoviendo la naturaleza de la responsabilidad social ambiental, pareciere que esto fuese retórico, aun cuando se le enseña al humano a consumir desmesuradamente, llegando al punto 
de una modificación en el patrón de conducta de uso, consumo y necesidad, que en ultimas termina afectando al ambiente.

\section{- La subjetividad en el espacio habitado}

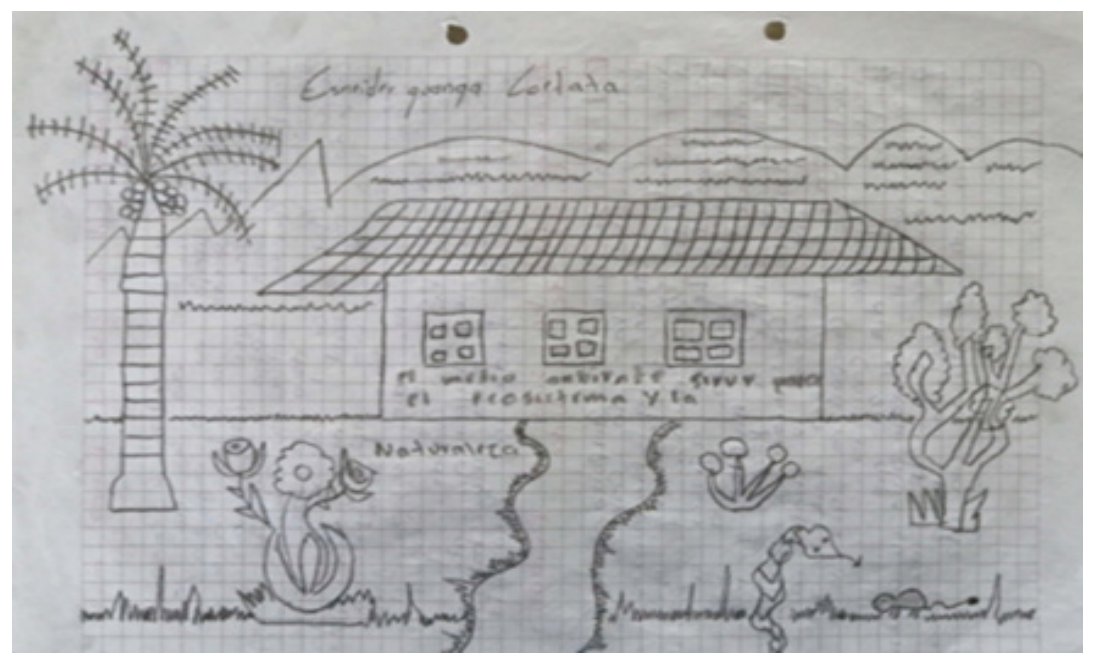

Figura 1. Espacio habitado. Construida por Esneider Guanga Coetata nota: del taller del significado del ambiente

Los seres humanos tienen la posibilidad de pensar lo que deseen frente al espacio que habitan. El contexto determina las condiciones de aprendizaje y comportamiento de la especie humana, Tabasura, (2009). En el grupo de participantes se evidenció, que hay una subjetividad desde el reconocimiento del ambiente como un factor inseparable del sujeto. En tiempos de la cuarentena, el significado y las percepciones del ambiente por parte del grupo focal experimentaron nuevas sensaciones, sonidos y se crearon conceptos relacionados con el ambiente y espacio que se habita, per se, a la dificultad de salud pública que se viene dando. La anterior imagen muestra, como la subjetividad está permeada por la construcción de un propio imaginario de ambiente, en tiempos de la pandemia, tal vez la percepción hubiese podido ser distinta.

Lo anterior conlleva a expresar, varias cosas, 1 . Hay una realidad vivida y sentida por el participante. 2. La percepción de espacio habitado durante la cuarentena incrementó la posibilidad de observar más elementos dentro del entorno a pesar de estar ligados a un sistema, con políticas que en algunas ocasiones desfavorecen a la comunidad y al sujeto, esta última trata de encontrar el significado por medio de la subjetividad y encuentro por aquello que está en el ambiente pero que no es visible o incluso explicable. Asimismo, el participante 3 afirma: 
"Sabemos que hay culebritas, animalitos y otros bichos, porque estamos en la zona rural, pero mantenemos tan ocupados con los procesos que la fundación nos brinda que cuando los vemos nos asombramos, pero ahora como hay poco ruido yo por lo menos, he visto animalitos que no los conocía" Participante 3.

La afirmación dada por el participante, menciona de forma implícita que la observación natural se desarrolla con el fin de comprender el significado de los elementos que configuran el entorno. En consideración, expone su preocupación sobre el ambiente en el que vive, el cual se encuentra con alteraciones que son visibles, pero posiblemente ignoradas.

\section{Transformación del entorno: de lo rural a lo urbano.}

Es pertinente mencionar que los crecimientos de las ciudades vienen a toda marcha. Esto genera una ruptura de la frontera entre lo rural y urbano, dando forma a nuevos espacios antrópicos como unidades de hábitats, Santos, Tellería, (2006). El crecimiento demográfico y social de algunas urbes están cambiando el imaginario del espacio habitado. El uso racional de los recursos naturales se agota. Asimismo, estuvieron en la palestra de la investigación, donde se reconoció que en ocasiones ellos consumen más de lo normal, pero tampoco hay un control sobre el consumo.

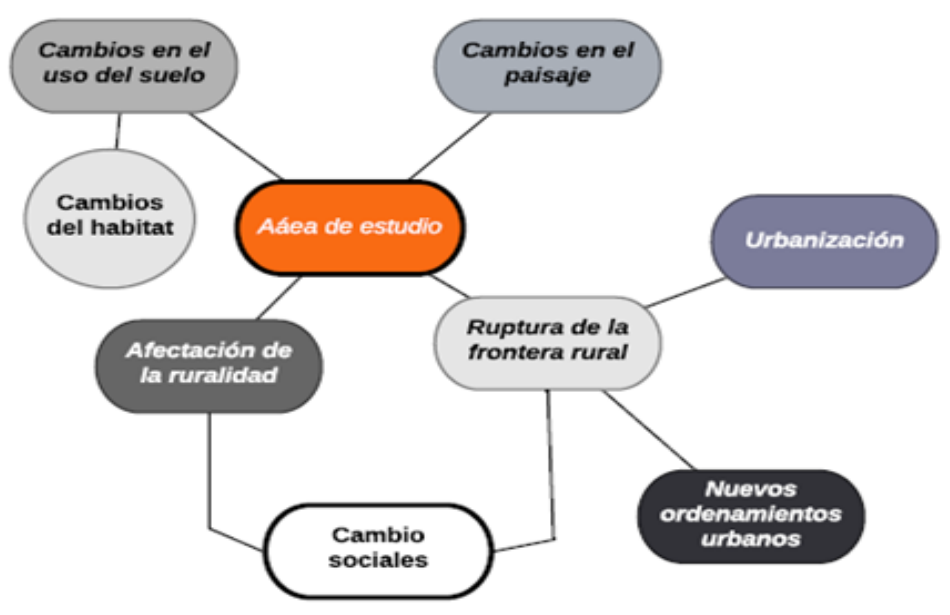

Figura 2. Notas: Dinámicas de entorno. De construcción propia, información obtenida de los diagramas de los participantes.

La construcción del anterior diagrama, evidencia como los participantes reflejan el cambio del espacio y como a partir del mismo empiezan a darse una serie de rupturas 
entre lo que es un espacio natural cuya configuración territorial es rural, el cual es transformado de forma urbana con características particulares en los cambios del paisaje. Asimismo, se puede apreciar como los participantes lograron prestar atención en varios de los componentes ambientales, los cuales siempre han visto alterados, y que se han ido recuperando, tomando como ejemplo: ver especies nuevas de aves para ellos en dicho espacio.

De otro lado, la percepción del ambiente, que los jóvenes desarrollaron permitió comprender como el confinamiento, terminó siendo un factor determinante para comprender el espacio, aumentando la integridad de los sujetos con todo aquello que está inmerso en la pervivencia.

\section{Conclusiones}

Los significados del ambiente se configuraron a través de los talleres de educación ambiental, las disertaciones, los dibujos y las entrevistas no estructuradas, permitiendo conocer como los participantes desarrollan la percepción del entorno, y como se adaptan a la pandemia COVID-19. Además, nace la subjetividad, ligada al ambiente como actor de análisis. La critica y reflexión sobre lo que se está haciendo en el contexto visto de forma micro, pero también se generan posturas desde lo macro, donde se acusa al sistema global como responsable de perder la capacidad de asombro y el olvido de lo que puede estar en el ambiente, pero por las dinámicas sociales y económicas no se permiten ver de otra forma.

Se entiende que la relación de los participantes con el medio, es una comunión de respeto, cuidado, pero uno de los temas que más los llevó a la reflexión de fomentar prácticas de protección, fue cuando entendieron que se encontraban en medio de una emergencia de salud pública. La realidad que viven los participantes, llega al punto de lo subjetivo, donde se permitieron evaluar su interacción con el ecosistema, es decir: como uno más del mismo.

El grupo focal, entendió que la transformación del espacio trajo consigo muchos impactos ambientales, pero en tiempos de la pandemia hubo reflexión sobre el entorno como un sistema proveedor de bienestar. El silencio producido por el confinamiento, posiblemente aumentó la percepción de todo aquello que el entorno ofrece al sujeto. La configuración de ambiente, se volatiliza por la saturación de la urbe, como un factor estresante dentro del medio natural. El espacio habitado se reconfigura en los jóvenes que hacen reflexiones, frente a sus propias sensaciones y percepciones que el ambiente ofrece que permanecieron de forma invisible, pero que se visibiliza en esta integración hombre naturaleza. 


\section{Lista de Referencias}

Abril, H. (2015). Ciudadanía, educación y complejidad: miradas desde la ecopedagogía, Dialogo Andino, 47: 95-104

Abril, L. M. (2021). Proceso de aprendizaje en la pandemia, Panorama, 15 (28), 188-205. Recuperado en: https://doi.org/10.15765/pnrm.v15i28.1823

Aguirre, R. (2012). Pensamiento narrativo y educación, Educere, 16 (53), 83-92, Mérida, Venezuela

Arias- Castilla, C. (2006). Enfoques teóricos sobre la, percepción que tienen las personas, Horizontes Pedagógicos, 8 (1), 9 - 22

Arias, V. N. \& Rincón, B. W. (2021). Educación básica y media durante el aislamiento social en la pandemia de covid-19: implicaciones para el derecho a la educación. Panorama, 15(29). Recuperado en: https://doi.org/10.15765/pnrm. v15i29.2622

Bracho- Mosquera, A. (2021). Análisis sociohistórico del concepto juventud. Revista Contacto, 1(1), 37-51. Recuperado a partir de https://www.revistas.up.ac.pa/ index.php/contacto/article/view/2206

Caruso, S. A. (2018). Crisis ambiental, pensamiento ambientalista y creación de áreas naturales protegidas en Argentina. Comunicaciones, 29, 74-102

Castro, O., R. (2004). Foucault y el saber educativo (primera parte: herramientas para una teoría crítica sobre la educación) Revista electrónica diálogos educativos. 4 (8), 40-50. Santiago de Chile- Chile

Díaz-Pérez, A. A., Cruz-Acevedo, A. A. (2020). Hacia un modelo de clases participativas de Ciencias Sociales en Educación Secundaria. Revista Electrónica de Conocimientos, Saberes y Prácticas, 3(2),79-86. DOI: https://doi.org/10.5377/ recsp.v3i2.10693

Domínguez, E., Herrera. J. (2011). Narrative inquiry in psychology: definition and functions. Psicología desde el Caribe, 30 (3), 620- 641.

Edel, N., R., Ramírez, G., M. (2006). Construyendo el significado del cuidado ambiental: un estudio de caso en educación secundaria REICE. Revista Iberoamericana sobre Calidad, Eficacia y Cambio en Educación, 4 (1),52-70. Madrid, España 


\section{INVESTIGACIÓN EDUCATIVA}

Eschenhagen, M. L (2007). Diversas consideraciones y aproximaciones a la noción de complejidad ambiental, Reflexión 10: 82-92

Espino, R, P., Olaguez, T, E., Davizon, C. Y. (2015). Análisis de la Percepción del Medio Ambiente de los Estudiantes de Ingeniería en Mecatrónica, Formación Universitaria, 8(4), 45-54: doi: 10.4067/So718-50062015000400006

Esteban, H., K. (2015). La teoría del poder de Foucault en el ámbito educativo, Horizonte de la Ciencia 5 (9), 127- 133.

Fernández, M, Y. (2008). ¿Por qué estudiar las percepciones ambientales? Una revisión de la literatura mexicana con énfasis en Áreas Naturales Protegidas, Espiral: Estudios sobre Estado y Sociedad, 15 (43), 179-200

García-Huidobro. (2016). Narrative as elicitation method and theoretical production in qualitative research, EMPIRIA. Revista de Metodología de Ciencias Sociales. 34, 155-178. DOI/empiria.34.2016.1652

Hernández- Castro, F. (2016). Teoría de la percepción. Ed. Instituto Tecnológico de Costa Rica, 1 edición.

Leff, E. (1998). Saber Ambiental: Sustentabilidad, Racionalidad, Complejidad, Poder, Ed. Siglo XXI, México.

Leff, E. (2004). Racionalidad ambiental la reapropiación social de la naturaleza, Ed. Siglo XXI, México

Leff, E. (2006). La ecología política en América Latina Un campo en construcción, CLACSO, Consejo Latinoamericano de Ciencias Sociales, recuperado en: http://bibliotecavirtual.clacso.org.ar/clacso/gt/20101002070402/3Leff.pdf

Leff, E. (2006). Pensamiento Ambiental Latinoamericano: Patrimonio de un Saber para la Sustentabilidad, Environmental Ethics, 34 (97)-112, DOI: 10.5840/ enviroethics201234Supplement58

Leff, E. (2007). La Complejidad Ambiental, Polis, Revista de la Universidad Bolivariana, 6 (16), 1-9 Universidad de Los Lagos Santiago, Chile

Leff, E. (2018). Racionalidad ambiental y diálogo de saberes: sentidos y senderos de un futuro sustentable, Desenvolvimento e Meio Ambiente, 7, 13-40

Martínez, M. E. (2014). Análisis crítico reflexivo sobre complejidad ambiental, ARJÉ Revista de Postgrado FACE-UC, 8 (14), 225-232 
Martínez-Castillo, R. (2010). La importancia de la educación ambiental ante la problemática actual Electrónica Educare, 24 (1), 97-111, Heredia, Costa Rica

Morales, J., G. (2016). El concepto "ambiente", una reflexión epistemológica sobre su uso y su estandarización en las ciencias ambientales, Electrónica Nova Scientia, 8 (2), 579-613, San Luis Potosí- México

Morin, E. (2001). Los siete saberes necesarios para la educación del futuro. Barcelona: Paidós.

Montoya-Ortega, Y. (2019). Gestión de la comunicación intercultural desde el diálogo de saberes y haceres. Revista Electrónica de Conocimientos, Saberes y Prácticas, 2(1), 8 -20. DOI: https://doi.org/10.5377/recsp.v2i1.8163

Novo, M., Murga, M. (2010). Educación ambiental y ciudadanía planetaria, Eureka sobre Enseñanza y Divulgación de las Ciencias, 7, 179-186 Cádiz, España

Osuna, C. J. (2021). La pandemia coronavirus Covid-19 y salud del medio ambiente, Educere, 25, (80), 259-262. Recuperado en: https://www.redalyc.org/articulo. oa? id $=35666280023$

Pereira, C., J. (2010). Consideraciones básicas del pensamiento Complejo de Edgar Morín, en la educación, Educare, 15 (1), 67-75

Plachot, G. B. (2021). Comunicación y docencia en la universidad doméstica-COVID19: ¿adaptación o reconfiguración? Revista Guatemalteca De Educación Superior, 4(2), 77-89. Recuperado en: https://doi.org/10.46954/revistages.v4i2.64

Quintero, M., Solarte, M. (2019). Las concepciones de ambiente inciden en el modelo de enseñanza de la educación ambiental, Entramado, 15(2), 130 - 147. Cali- Colombia

Rekalde, I., Vizcarra, M., Macazaga, A. (2014). La Observación Como Estrategia De Investigación Para Construir Contextos De Aprendizaje Y Fomentar Procesos Participativos Educación XX1, 17 (1), 201-220 Madrid, España

Rodríguez, S., R. (2010). Algunas reflexiones sobre el concepto de ambiente en las ciencias sociales contemporáneas, Provincia, 23, 113-125, Mérida, Venezuela

Rosales, S. J. (2018). La teoría de la percepción en la Filosofía del Entendimiento de Andrés Bello, Anales del Seminario de Historia de la Filosofía, 36:(1), 127-145. Doi: 10.5209/ASHF.63365 


\section{INVESTIGACIÓN EDUCATIVA}

Santos, T., Tellería, J.L. (2006). Pérdida y fragmentación del hábitat: efecto sobre la conservación de las especies, Ecosistemas, 15 (2), 3-12.

Tabasura, A. I. (2009). Augusto Ángel Maya: aportes de caldas al pensamiento y movimiento ambiental colombiano, Luna Azúl, 28: 57-67 